\title{
OPERATOR JENSEN'S INEQUALITY FOR OPERATOR SUPERQUADRATIC FUNCTIONS
}

\author{
MOHAMMAD W. ALOMARI
}

\begin{abstract}
In this work, an operator superquadratic function (in operator sense) for positive Hilbert space operators is defined. Several examples with some important properties together with some observations which are related to the operator convexity are pointed out. Equivalent statements of a non-commutative version of Jensen's inequality for operator superquadratic function are established. A generalization of the main result to any positive unital linear map is also provided.
\end{abstract}

\section{INTRODUCTION}

Let $\mathscr{B}(\mathscr{H})$ be the Banach algebra of all bounded linear operators defined on a complex Hilbert space $(\mathscr{H} ;\langle\cdot, \cdot\rangle)$ with the identity operator $1_{\mathscr{H}}$ in $\mathscr{B}(\mathscr{H})$. A bounded linear operator $A$ defined on $\mathscr{H}$ is selfadjoint if and only if $\langle A x, x\rangle \in \mathbb{R}$ for all $x \in \mathscr{H}$. The spectrum of an operator $A$ is the set of all $\lambda \in \mathbb{C}$ for which the operator $\lambda I-A$ does not have a bounded linear operator inverse, and is denoted by $\operatorname{sp}(A)$. Consider the real vector space $\mathscr{B}(\mathscr{H})_{s a}$ of self-adjoint operators on $\mathscr{H}$ and its positive cone $\mathscr{B}(\mathscr{H})^{+}$of positive operators on $\mathscr{H}$. Also, $\mathscr{B}(\mathscr{H})_{s a}^{I}$ denotes the convex set of bounded self-adjoint operators on the Hilbert space $\mathscr{H}$ with spectra in a real interval $I$. A partial order is naturally equipped on $\mathscr{B}(\mathscr{H})_{s a}$ by defining $A \leq B$ if and only if $B-A \in \mathscr{B}(\mathscr{H})^{+}$. We write $A>0$ to mean that $A$ is a strictly positive operator, or equivalently, $A \geq 0$ and $A$ is invertible. When $\mathscr{H}=\mathbb{C}^{n}$, we identify $\mathscr{B}(\mathscr{H})$ with the algebra $\mathfrak{M}_{n \times n}$ of $n$-by- $n$ complex matrices. Then, $\mathfrak{M}_{n \times n}^{+}$is just the cone of $n$-by- $n$ positive semidefinite matrices.

A linear map is defined to be $\Phi: \mathscr{B}(\mathscr{H}) \rightarrow \mathscr{B}(\mathscr{K})$ which preserves additivity and homogeneity, i.e., $\Phi\left(\lambda_{1} A+\lambda_{2} B\right)=\lambda_{1} \Phi(A)+\lambda_{2} \Phi(B)$ for any $\lambda_{1}, \lambda_{2} \in \mathbb{C}$ and $A, B \in \mathscr{B}(\mathscr{H})$. The linear map is positive $\Phi: \mathscr{B}(\mathscr{H}) \rightarrow \mathscr{B}(\mathscr{K})$ if it preserves the operator order, i.e., if $A \in \mathscr{B}^{+}(\mathscr{H})$ then $\Phi(A) \in \mathscr{B}^{+}(\mathscr{K})$, and in this case we write $\mathcal{B}[\mathscr{B}(\mathscr{H}), \mathscr{B}(\mathscr{K})]$. Obviously, a positive linear map $\Phi$ preserves the order relation, namely $A \leq B \Longrightarrow \Phi(A) \leq \Phi(B)$ and preserves the adjoint operation $\Phi\left(A^{*}\right)=\Phi(A)^{*}$. Moreover, $\Phi$ is said to be unital if it preserves the identity operator, in this case we write $\mathcal{B}_{n}[\mathscr{B}(\mathscr{H}), \mathscr{B}(\mathscr{K})]$.

A linear map $\Phi: \mathscr{B}(\mathscr{H}) \rightarrow \mathscr{B}(\mathscr{K})$ induces another map

$$
\mathrm{id} \otimes \Phi: \mathbb{C}^{k \times k} \otimes \mathscr{B}(\mathscr{H}) \rightarrow \mathbb{C}^{k \times k} \otimes \mathscr{B}(\mathscr{K}),
$$

in a natural way. If $\mathbb{C}^{k \times k} \otimes \mathscr{B}(\mathscr{H})$ is identified with the $C^{*}$-algebra $\mathscr{B}^{k \times k}(\mathscr{H})$ of $k \times k$-matrices with entries in $\mathscr{B}(\mathscr{H})$ then id $\otimes \Phi$ act as:

$$
\left(\begin{array}{ccc}
A_{11} & \cdots & A_{1 k} \\
\vdots & \ddots & \vdots \\
A_{k 1} & \cdots & A_{k k}
\end{array}\right) \mapsto\left(\begin{array}{ccc}
\Phi\left(A_{11}\right) & \cdots & \Phi\left(A_{1 k}\right) \\
\vdots & \ddots & \vdots \\
\Phi\left(A_{k 1}\right) & \cdots & \Phi\left(A_{k k}\right)
\end{array}\right) .
$$

We say that $\Phi$ is $k$-positive if id $\otimes \Phi$ is a positive map, and $\Phi$ is called completely positive if $\Phi$ is $k$-positive for all $k$.

1.1. Superquadratic functions. A function $f: J \rightarrow \mathbb{R}$ is called convex iff

$$
f(t \alpha+(1-t) \beta) \leq t f(\alpha)+(1-t) f(\beta),
$$

for all points $\alpha, \beta \in J$ and all $t \in[0,1]$. If $-f$ is convex then we say that $f$ is concave. Moreover, if $f$ is both convex and concave, then $f$ is said to be affine.

Date: December 15, 2019.

2010 Mathematics Subject Classification. 47A63, 47A56.

Key words and phrases. Operator supequadratic, Operator convex, Selfadjoint, Jensen inequality. 
Geometrically, for two point $(x, f(x))$ and $(y, f(y))$ on the graph of $f$ are on or below the chord joining the endpoints for all $x, y \in I, x<y$. In symbols, we write

$$
f(t) \leq \frac{f(y)-f(x)}{y-x}(t-x)+f(x)
$$

for any $x \leq t \leq y$ and $x, y \in J$.

Equivalently, given a function $f: J \rightarrow \mathbb{R}$, we say that $f$ admits a support line at $x \in J$ if there exists a $\lambda \in \mathbb{R}$ such that

$$
f(t) \geq f(x)+\lambda(t-x)
$$

for all $t \in J$.

The set of all such $\lambda$ is called the subdifferential of $f$ at $x$, and it's denoted by $\partial f$. Indeed, the subdifferential gives us the slopes of the supporting lines for the graph of $f$. So that if $f$ is convex then $\partial f(x) \neq \emptyset$ at all interior points of its domain.

From this point of view Abramovich et al. [2] extend the above idea for what they called superquadratic functions. Namely, a function $f:[0, \infty) \rightarrow \mathbb{R}$ is called superquadratic provided that for all $x \geq 0$ there exists a constant $C_{x} \in \mathbb{R}$ such that

$$
f(t) \geq f(x)+C_{x}(t-x)+f(|t-x|)
$$

for all $t \geq 0$. We say that $f$ is subquadratic if $-f$ is superquadratic. Thus, for a superquadratic function we require that $f$ lie above its tangent line plus a translation of $f$ itself. If $f$ is differentiable and satisfies $f(0)=f^{\prime}(0)=0$, then one sees easily that the $C_{x}$ appearing in the definition is necessarily $f^{\prime}(x),($ see $[1])$.

Prima facie, superquadratic function looks to be stronger than the convex function itself but if $f$ takes negative values then it maybe considered as a weaker function. Therefore, if $f$ is superquadratic and nonnegative, then $f$ is convex and increasing [2] (see also [4]).

Moreover, the following result holds for superquadratic function.

Lemma 1. [2] Let $f$ be superquadratic function. Then

(1) $f(0) \leq 0$

(2) If $f$ is differentiable and $f(0)=f^{\prime}(0)=0$, then $C_{x}=f^{\prime}(x)$ for all $x \geq 0$.

(3) If $f(x) \geq 0$ for all $x \geq 0$, then $f$ is convex and $f(0)=f^{\prime}(0)=0$.

The next result gives a sufficient condition when convexity (concavity) implies super(sub)quaradicity.

Lemma 2. [2] If $f^{\prime}$ is convex (concave) and $f(0)=f^{\prime}(0)=0$, then is super(sub)quadratic. The converse of is not true.

Remark 1. In general, non-negative subquadratic functions does not imply concavity. In other words, there exists a subquadratic function which is convex. For example, $f(x)=x^{p}, x \geq 0$ and $1 \leq p \leq 2$ is subquadratic and convex.

Among others, Abramovich et al. [2] proved that the inequality

$$
f\left(\int \varphi d \mu\right) \leq \int f(\varphi(s))-f\left(\left|\varphi(s)-\int \varphi d \mu\right|\right) d \mu(s)
$$

holds for all probability measures $\mu$ and all nonnegative, $\mu$-integrable functions $\varphi$ if and only if $f$ is superquadratic. For more details the reader may refer to [4], [5], and [22].

1.2. Operator convexity and Jensen inequality. Let $f$ be a real-valued function defined on $J$. A $k$-th order divided difference of $f$ at distinct points $x_{0}, \cdots, x_{k}$ in $J$ may be defined recursively by

$$
\begin{aligned}
{\left[x_{i}\right] f } & =f\left(x_{i}\right) \\
{\left[x_{0}, x_{1}, \ldots, x_{k}\right] f } & =\frac{\left[x_{1}, \ldots, x_{k}\right] f-\left[x_{0}, \ldots, x_{k-1}\right] f}{x_{k}-x_{0}} .
\end{aligned}
$$


For instance, the first 3-divided differences are given as follows:

$$
\begin{aligned}
& {\left[x_{0}\right] f=f\left(x_{0}\right) \quad \text { if } \quad k=0,} \\
& {\left[x_{0}, x_{1}\right] f=\frac{\left[x_{1}\right] f-\left[x_{0}\right] f}{x_{1}-x_{0}} \quad \text { if } \quad k=1,} \\
& {\left[x_{0}, x_{1}, x_{2}\right] f=\frac{\left[x_{1}, x_{2}\right] f-\left[x_{0}, x_{1}\right] f}{x_{2}-x_{0}} \quad \text { if } \quad k=2 .}
\end{aligned}
$$

A function $f: J \rightarrow \mathbb{R}$ is said to be matrix monotone of degree $n$ or $n$-monotone, if for every $A, B \in \mathfrak{M}_{n \times n}$, it is true that $A \leq B \Longleftrightarrow f(A) \leq(B)$. Similarly, $f$ is said to be operator monotone If $f$ is $n$-monotone for all $n \in \mathbb{N}$. Also, $f$ is called operator convex if it is matrix convex ( $n$-convex for all $n$ ); i.e., if for every pair of selfadjoint operators $A, B \in \mathfrak{M}_{n \times n}$ we have

$$
f(\lambda A+(1-\lambda) B) \leq \lambda f(A)+(1-\lambda) f(B)
$$

for all $\lambda \in[0,1]$. If the inequality is reversed then $f$ is called operator concave. In case we have general Hilbert space $\mathscr{H}$, the above definition holds for every pair of bounded selfadjoint operators $A$ and $B$ in $\mathscr{B}(\mathscr{H})$, whose spectra ontianed in $J$. For more details see [23] and the recent survey [14].

In 1955, Bendat and Sherman [13] have shown that $f$ is operator convex on the open interval $(-1,1)$ if and only if it has a (unique) representation

$$
f(t)=\beta_{0}+\beta_{1} t+\frac{1}{2} \beta_{2} \int_{-1}^{1} \frac{t^{2}}{1-\alpha t} d \mu(t)
$$

for $\beta_{2} \geq 0$ and some probability measure $\mu$ on $[-1,1]$ (it could be Borel measure). In particular, $f$ must be analytic with $f(0)=\beta_{0}, f^{\prime}(0)=\beta_{1}$ and $f^{\prime \prime}(0)=\beta_{2}$.

We recall that the celebrated Löwner-Heinz inequality reads that:

Lemma 3. Let $A, B \in \mathscr{B}(\mathscr{H})^{+}$such that $A \geq B$, then $A^{r} \geq B^{r}$ for all $r \in[0,1]$.

On the other hand the mapping $t \mapsto t^{p}(p>1)$ is not operator monotone, for more details see [10], [17] and [19].

The classical Jensen's inequality for reals states that

$$
f\left(\sum_{j=1}^{n} \lambda_{j} x_{j}\right) \leq \sum_{j=1}^{n} \lambda_{j} f\left(x_{j}\right)
$$

valid for all real valued convex function $f$ defined on $[m, M]$, for every $x_{1}, \cdots, x_{n} \in[m, M]$ and every positive real numbers $\lambda_{j}(1 \leq j \leq n)$ such that $\sum_{j=1}^{n} \lambda_{j}=1$.

The inequality (1.5) would be rephrases under matrix situation by putting

$$
A=\left(\begin{array}{ccc}
x_{1} & & 0 \\
& \ddots & \\
0 & & x_{n}
\end{array}\right) \quad \text { and } \quad x=\left(\begin{array}{c}
\sqrt{\lambda_{1}} \\
\vdots \\
\sqrt{\lambda_{n}}
\end{array}\right)
$$

then the classical Jensen inequality (1.5) is expressed as

$$
f(\langle A x, x\rangle) \leq(f(A) x, x)
$$

which is one of the operator version of the classical Jensen's inequality, see [17].

Kadison [21] established his famous non-commutative version of the previous inequality where he proved that for every selfadjoint matrix $A$ the inequality

$$
\Phi^{2}(A) \leq \Phi\left(A^{2}\right)
$$

for every positive unital linear map $\Phi: \mathfrak{M}_{n \times n}(\mathbb{C}) \rightarrow \mathfrak{M}_{k \times k}(\mathbb{C})$.

This inequality was generalized later by Davis in [16], where he obtained that this is true when $f$ is a matrix convex function and $\Phi$ is completely positive; i.e.,

$$
f(\Phi(A)) \leq \Phi(f(A))
$$


The latter restriction about complete positivity of $\Phi$ was removed by Choi [15] who proved that (1.7) remains valid for all positive unital linear maps provided $f$ is matrix convex.

Another noncommutative operator version of the classical Jensen's inequality under the situation that

$$
A=\left(\begin{array}{ccc}
x_{1} & & 0 \\
& \ddots & \\
0 & & x_{n}
\end{array}\right) \quad \text { and } \quad V=\left(\begin{array}{ccc}
\sqrt{\lambda_{1}} & \cdots & 0 \\
\vdots & \ddots & \\
\sqrt{\lambda_{n}} & & 0
\end{array}\right)
$$

then the classic Jensen's inequality is expressed as

$$
f\left(V^{*} A V\right) \leq V^{*} f(A) V
$$

The inequality (1.9) was proved by Davis in [16] for all $A \in \mathscr{B}(\mathscr{H})$ and every isometry $C$. However, a more informative version was extended by Hansen-Pedersen [19] as follows:

Theorem 1. Let $\mathscr{H}$ and $\mathscr{K}$ be Hilbert space. Let $f$ be a real valued continuous function on an interval I. Let $A$ and $A_{j}$ be selfadjoint operators on $\mathscr{H}$ with spectra contained in $I(j=1,2, \cdots, k)$. Then the following conditions are mutually

(1) $f$ is operator convex on $I$ and $f(0) \leq 0$.

(2) $f\left(C^{*} A C\right) \leq C^{*} f(A) C$, for every $A \in \mathscr{B}(\mathscr{H})$ and contraction $C \in \mathscr{B}(\mathscr{H})$; i.e., $C^{*} C \leq 1_{\mathscr{K}}$.

(3) $f\left(\sum_{j=1}^{n} C_{j}^{*} A_{j} C_{j}\right) \leq \sum_{j=1}^{n} C_{j}^{*} f\left(A_{j}\right) C_{j}$, for all $A_{j} \in \mathscr{B}(\mathscr{H})$ and $C_{j} \in \mathscr{B}(\mathscr{H})$ with $\sum_{j=1}^{n} C_{j}^{*} C_{j} \leq 1_{\mathscr{H}}$, $(j=1,2, \cdots, k)$.

(4) $f(P A P) \leq P f(A) P$, for every $A \in \mathscr{B}(\mathscr{H})$ and projection $P$.

Here we give some popular examples of operator convex and concave function [14].

(1) For each $p \in[0,1], t^{p} \quad$ is operator concave on $[0, \infty)$.

(2) The function $t \log t$ is operator convex on $[0, \infty)$.

This work is organized as follows: after this introduction; in Section 2, the operator superquadratic functions for positive Hilbert space operators are introduced and elaborated. Several examples with some important properties together with some observations related to operator convexity are pointed out. In Section 3, A Jensen type inequality is proved. Equivalent statements of a non-commutative version in of Jensen's inequality for operator superquadratic are also established. Finally, several trace inequalities for superquadratic functions (in ordinary sense) are provided as well.

\section{Operator SUPERQUADRATIC FUNCTION}

Definition 1. Let $I=[0, M] \subseteq[0, \infty) . \quad$ A real valued continuous function $f(t)$ on an interval $I$ is said to be operator superquadratic function if

$$
f(\alpha A+(1-\alpha) B)
$$

$$
\leq \alpha[f(A)-f((1-\alpha)|A-B|)]+(1-\alpha)[f(B)-f(\alpha|A-B|)]
$$

holds for all $\alpha \in[0,1]$ and for every positive operators $A$ and $B$ on a Hilbert space $\mathscr{H}$ whose spectra are contained in $I \subset[0, \infty)$. We say that $f$ is operator subquadratic function if $-f$ is operator superquadratic function. Moreover, if the equality holds in (2.1), in this case we say that $f$ is operator quadratic function.

It's convenient to note that; if $f$ satisfies (2.1), then with $A=x$ and $B=y$ (two positive scalars) one can obtain the Jensen inequality for superquadratic functions and if $f$ is continuous (which is necessary to define an operator functions), then (1.4) would imply that $f$ is superquadratic function. Thus, we observe that:

Corollary 1. If $f$ is an operator superquadratic function then $f$ is a real superquadratic function. 
Let $f(t)=\alpha t+\beta$, then $f$ is operator subquadratic on every bounded interval for all $\alpha, \beta \geq 0$. Indeed, we have

$$
\begin{aligned}
& f\left(\frac{A+B}{2}\right)+f\left(\frac{|A-B|}{2}\right)-\frac{f(A)+f(B)}{2} \\
& =\left[\alpha \frac{A+B}{2}+\beta\right]+\left[\alpha \frac{|A-B|}{2}+\beta\right]-\frac{\alpha A+\beta+\alpha B+\beta}{2} \\
& =\alpha \frac{|A-B|}{2}+\beta \geq 0 .
\end{aligned}
$$

Moreover, $g(t)=-f(t)$ is operator superquadratic.

One can easily seen that the function $t \mapsto t^{3}$ is not operator superquadratic nor operator subquadratic function. Simply, assume $f(t)=t^{3}, t \in[0, \infty)$ and let

$$
A=\left(\begin{array}{ll}
2 & 1 \\
1 & 1
\end{array}\right) \quad \text { and } \quad B=\left(\begin{array}{ll}
1 & 0 \\
0 & 0
\end{array}\right)
$$

then,

$$
\frac{A^{3}+B^{3}}{2}-\left(\frac{A+B}{2}\right)^{3}-\left(\frac{|A-B|}{2}\right)^{3}=\frac{1}{4}\left(\begin{array}{ll}
9 & 7 \\
7 & 5
\end{array}\right) \underset{\ngtr}{\underset{\ddagger}{\not} 0 .}
$$

However, the map $t \mapsto t^{2}$ is non-negative operator convex on $(0, \infty)$ and it is also operator superquadratic on $(0, \infty)$. Indeed, by $(2.1)$ we have

$$
\begin{aligned}
& (\alpha A+(1-\alpha) B)^{2} \leq \alpha A^{2}+(1-\alpha) B^{2}-\alpha(1-\alpha)^{2}|A-B|^{2}-(1-\alpha) \alpha^{2}|A-B|^{2} \\
& \Leftrightarrow \alpha^{2} A^{2}+(1-\alpha)^{2} B^{2}+\alpha(1-\alpha)(A B+B A) \leq \alpha A^{2}+(1-\alpha) B^{2} \\
& \quad-\left[\alpha(1-\alpha)^{2}+(1-\alpha) \alpha^{2}\right](A-B)^{2} \\
& \Leftrightarrow \alpha(\alpha-1) A^{2}+\alpha(\alpha-1) B^{2}+\alpha(\alpha-1)(A B+B A) \leq \alpha(\alpha-1)(A-B)^{2} \\
& \Leftrightarrow \alpha(\alpha-1)(A+B)^{2} \leq \alpha(\alpha-1)(A-B)^{2} \\
& \Leftrightarrow(A+B)^{2} \geq(A-B)^{2} \quad \text { for } \alpha(\alpha-1)<0 \\
& \Leftrightarrow|A+B| \geq|A-B| \quad g(t)=\sqrt{t} \text { is operator monotone }
\end{aligned}
$$

which is true since $A, B>0$, and this proves that $t^{2}$ is operator superquadratic function.

From the definition of operator superquadratic function we have

$$
f(\alpha A+(1-\alpha) B) \leq \alpha[f(A)-f((1-\alpha)|A-B|)]+(1-\alpha)[f(B)-f(\alpha|A-B|)]
$$

for any arbitrary positive operators $A, B \in \mathscr{B}(\mathscr{H})$ and each $\alpha \in[0,1]$.

In particular, by setting $B=\langle A x, x\rangle 1_{\mathscr{H}}$ in (2.1) we have

$$
\begin{aligned}
f\left(\alpha A+(1-\alpha)\langle A x, x\rangle 1_{\mathscr{H}}\right) \leq \alpha\left[f(A)-f\left((1-\alpha)\left|A-\langle A x, x\rangle 1_{\mathscr{H}}\right|\right)\right] \\
+(1-\alpha)\left[f(\langle A x, x\rangle)-f\left(\alpha\left|A-\langle A x, x\rangle 1_{\mathscr{H}}\right|\right)\right]
\end{aligned}
$$

for each positive operator $A \in \mathscr{B}(\mathscr{H})$ and all $\alpha \in[0,1]$.

From this point of view (2.3), Kian early in [24] and then jointly with Dragomir in [25] proved a finite dimensional operator version of Jensen's inequality for superquadratic functions (in ordinary sense) under the interpretation that for $A=\left(\begin{array}{ll}a & 0 \\ 0 & b\end{array}\right)$ and $x=\left(\begin{array}{c}\sqrt{\lambda} \\ \sqrt{1-\lambda}\end{array}\right)$, then we have $\langle A x, x\rangle=\lambda a+(1-\lambda) b$ if follows that

$$
|A-\langle A x, x\rangle|=\left(\begin{array}{cc}
(1-\lambda)|a-b| & 0 \\
0 & \lambda|a-b|
\end{array}\right) .
$$

Therefore, as a matrix Jensen inequality for a superquadratic function $f:[0, \infty) \rightarrow \mathbb{R}$ we have

$$
f(\langle A x, x\rangle) \leq\langle f(A) x, x\rangle+\langle f(|A-\langle A x, x\rangle|) x, x\rangle .
$$

This result was generalized for positive unital linear maps, as follows: 
Theorem 2. ([25], [7]) Let $A \in \mathscr{B}(\mathscr{H})$ be a positive operator and $\Phi: \mathscr{B}(\mathscr{H}) \rightarrow \mathscr{B}(\mathscr{K})$ be a positive unital linear map. If $f:[0, \infty) \rightarrow \mathbb{R}$ is super(sub)quadratic function, then we have

$$
\langle\Phi(f(A)) x, x\rangle \geq(\leq) f(\langle\Phi(A) x, x\rangle)+\left\langle\Phi\left(f\left(\left|A-\langle\Phi(A) x, x\rangle 1_{\mathscr{H}}\right|\right)\right) x, x\right\rangle
$$

for every $x \in \mathscr{K}$ with $\|x\|=1$.

The above inequality and other consequences were proved later by the first author of this paper in [7] where different approach is used.

Proposition 1. Let $f$ be an operator superquadratic function on $I$. Then

(1) $f(0) \leq 0$.

(2) If $f$ is non-negative, then $f$ is operator convex and $f(0)=0$.

Proof. (1) Setting $A=B=0$ in (2.3) we ge that $f(0) \leq 0$.

(2) Since $f$ is continuous and non-negative, then from (2.3) we have

$$
\begin{aligned}
f(\alpha A+(1-\alpha) B) & \leq \alpha[f(A)-f((1-\alpha)|A-B|)]+(1-\alpha)[f(B)-f(\alpha|A-B|)] \\
& \leq \alpha f(A)+(1-\alpha) f(B)
\end{aligned}
$$

which means that $f$ is operator convex. To show that $f(0)=0$, we have by part $(1) f(0) \leq 0$ and by assumption $f(x)$ is non-negative i.e., $f(x) \geq 0$ for all $x \in I$. In particular, $f(0) \geq 0$. Thus, $f(0)=0$.

Example 1. Let $f(t)=t^{-1}$, then $f$ is non-negative operator convex on $(0, \infty)$. However, $f$ is not operator superquadratic function on $(0, \infty)$. For instance, let

$$
A=\left(\begin{array}{ll}
3 & 0 \\
0 & 1
\end{array}\right) \quad \text { and } \quad B=\left(\begin{array}{ll}
1 & 0 \\
0 & 2
\end{array}\right)
$$

Applying (??) for $f(t)=t^{-1}$, we get

$$
\begin{aligned}
\frac{A^{-1}+B^{-1}}{2}-\left(\frac{A+B}{2}\right)^{-1}-\left(\frac{|A-B|}{2}\right)^{-1} & =\frac{1}{12}\left(\begin{array}{cc}
8 & 0 \\
0 & 9
\end{array}\right)-\frac{2}{12}\left(\begin{array}{cc}
3 & 0 \\
0 & 4
\end{array}\right)-\frac{6}{12}\left(\begin{array}{cc}
1 & 0 \\
0 & 2
\end{array}\right) \\
& =\frac{1}{12}\left(\begin{array}{cc}
-4 & 0 \\
0 & -11
\end{array}\right)<0
\end{aligned}
$$

Proposition 2. Let $f$ be a real valued continuous function defined on an interval $[0, \infty)$. If $f$ is operator convex and non-positive then $f$ is operator superquadratic function.

Proof. Since $f$ is operator convex, then

$$
\frac{f(A)+f(B)}{2}-f\left(\frac{A+B}{2}\right) \geq 0 .
$$

But also $f$ is non-positive, so that

$$
\frac{f(A)+f(B)}{2}-f\left(\frac{A+B}{2}\right)-f\left(\frac{|A-B|}{2}\right) \geq-f\left(\frac{|A-B|}{2}\right) \geq 0
$$

which means that $f$ is operator superquadratic function.

Example 2. Let $f(t)=t \log (t), t \in[0, \infty)$ it well known that $f$ operator convex. Clearly, $f$ is negative for all $t \in(0,1) \subseteq[0, \infty)$. Therefore, $f(t)=t \log (t)$ is operator superquadratic function for all $t \in(0,1)$.

Proposition 3. Let $f$ be a real valued continuous function defined on an interval $[0, \infty)$. If $f$ is operator concave and non-negative then $f$ is operator subquadratic.

Proof. Since $f$ is operator concave, then

$$
f\left(\frac{A+B}{2}\right)-\frac{f(A)+f(B)}{2} \geq 0 .
$$

But also $f$ is non-negative, so that

$$
f\left(\frac{A+B}{2}\right)-\frac{f(A)+f(B)}{2}+f\left(\frac{|A-B|}{2}\right) \geq f\left(\frac{|A-B|}{2}\right) \geq 0
$$


which means that $f$ is operator subquadratic.

Example 3. Let $f:(0, \infty) \rightarrow(0, \infty)$, given by $f(t)=t^{r}, r \in[0,1]$. Then $f$ is operator subquadratic on $(0, \infty)$. But $f$ is also operator concave, so that

$$
\frac{A^{r}+B^{r}}{2} \leq\left(\frac{A+B}{2}\right)^{r} \leq\left(\frac{A+B}{2}\right)^{r}+\left(\frac{|A-B|}{2}\right)^{r}
$$

which means $f$ is operator subquadratic on $(0, \infty)$.

\section{Operator Jensen's inequality}

In order to prove our results we need the following Lemmas:

Lemma 4. ([17]) If $A \in \mathscr{B}(\mathscr{H})$ is selfadjoint and $U$ is unitary, i.e. $U^{*} U=U U^{*}=1 \mathscr{H}$, then $f\left(U^{*} A U\right)=$ $U^{*} f(A) U$ for every $f$ continuous on the $\operatorname{sp}(A)$.

Lemma 5. ([18]) Define a unitary matrix $E_{n}=\operatorname{diag}\left(\xi, \xi^{2}, \cdots, \xi^{n-1}, 1\right)$ in $\mathfrak{M}_{n}(\mathbb{C}) \subset \mathscr{B}\left(\mathscr{H}^{n}\right)$, where $\xi=\exp \left(\frac{2 \pi i}{n}\right)$. Then for each element $A=\left(a_{i j}\right) \in \mathscr{B}\left(\mathscr{H}^{n}\right)$ we have

$$
\frac{1}{n} \sum_{k=1}^{n} E^{-k} A E^{k}=\operatorname{diag}\left(a_{11}, a_{22}, \cdots, a_{n n}\right)
$$

Lemma 6. ([18]) Let $P$ denote the projection in $\mathfrak{M}_{n}$ given by $P_{i j}=n^{-1}$ for all $i$ and $j$, so that $P$ is the projection of rank one on the subspace spanned by the vector $\xi+\xi^{2}+\cdots+\xi^{n}$ in $\mathbb{C}^{n}$, where $\xi, \xi^{2}, \cdots, \xi^{n}$ are the standard basis vectors. Then with $E$ as in Lemma 5 we obtain the pairwise orthogonal projections $P_{k}=E^{-k} P E^{k}$, for $1 \leq k \leq n$, with $\sum_{k=1}^{n} P_{k}=1$.

To establish our main first result we need the following primary result.

Lemma 7. Let $w_{1}, \ldots, w_{n}$ be positive real numbers such that $W_{n}=\sum_{k=1}^{n} w_{k}$ and let $A_{1}, \cdots, A_{n}$ be positive operators of a Hilbert space $\mathscr{B}(\mathscr{H})$ with spectra contained in a real interval $I$. If $f$ is operator superquadratic function on $I$, then

$$
f\left(\frac{1}{W_{n}} \sum_{k=1}^{n} w_{k} A_{k}\right) \leq \sum_{k=1}^{n} \frac{w_{k}}{W_{n}} f\left(A_{k}\right)-\sum_{k=1}^{n} \frac{w_{k}}{W_{n}} f\left(\left|A_{k}-\sum_{j=1}^{n} \frac{w_{j}}{W_{n}} A_{j}\right|\right)
$$

In particular useful case, for $w_{k}=1$ for all $1 \leq k \leq n$, we have

$$
f\left(\frac{1}{n} \sum_{k=1}^{n} A_{k}\right) \leq \frac{1}{n} \sum_{k=1}^{n} f\left(A_{k}\right)-\frac{1}{n} \sum_{k=1}^{n} f\left(\left|A_{k}-\frac{1}{n} \sum_{j=1}^{n} A_{j}\right|\right)
$$

Proof. Assume $f$ is operator superquadratic. If $n=2$, then the inequality (3.1) reduces to (2.1) with $\alpha=\frac{w_{1}}{W_{2}}$ and $1-\alpha=\frac{w_{2}}{W_{2}}$. Let us suppose that inequality (3.1) holds for $n-1$. Then for $n$-tuples $\left(A_{1}, \cdots, A_{n}\right)$ and 
$\left(w_{1}, \cdots, w_{n}\right)$, we have

$$
\begin{aligned}
& f\left(\frac{1}{W_{n}} \sum_{k=1}^{n} w_{k} A_{k}\right)= f\left(\frac{w_{n}}{W_{n}} A_{n}+\sum_{k=1}^{n-1} \frac{w_{k}}{W_{n}} A_{k}\right) \\
&= f\left(\frac{w_{n}}{W_{n}} A_{n}+\frac{W_{n-1}}{W_{n}} \sum_{k=1}^{n-1} \frac{w_{k}}{W_{n-1}} A_{k}\right) \\
& \leq \frac{w_{n}}{W_{n}}\left[f\left(A_{n}\right)-f\left(\frac{W_{n-1}}{W_{n}}\left|A_{n}-\frac{1}{W_{n-1}} \sum_{k=1}^{n-1} w_{k} A_{k}\right|\right)\right] \\
&+\frac{W_{n-1}}{W_{n}}\left[f\left(\sum_{k=1}^{n-1} \frac{w_{k}}{W_{n-1}} A_{k}\right)-f\left(\frac{w_{n}}{W_{n}}\left|A_{n}-\frac{1}{W_{n-1}} \sum_{k=1}^{n-1} w_{k} A_{k}\right|\right)\right] \\
&= \frac{w_{n}}{W_{n}} f\left(A_{n}\right)+\frac{W_{n-1}}{W_{n}} f\left(\sum_{k=1}^{n-1} \frac{w_{k}}{W_{n-1}} A_{k}\right) \\
&-\frac{w_{n}}{W_{n}} f\left(\frac{W_{n-1}}{W_{n}}\left|A_{n}-\frac{1}{W_{n-1}} \sum_{k=1}^{n-1} w_{k} A_{k}\right|\right) \\
& \quad-\frac{W_{n-1}}{W_{n}} f\left(\frac{w_{n}}{W_{n}}\left|A_{n}-\frac{1}{W_{n-1}} \sum_{k=1}^{n-1} w_{k} A_{k}\right|\right)
\end{aligned}
$$

and this is exactly equivalent to write, for any $1 \leq m \leq n$

$$
f\left(\frac{1}{W_{m}} \sum_{k=1}^{m} w_{k} A_{k}\right) \leq \sum_{k=1}^{m} \frac{w_{k}}{W_{m}} f\left(A_{k}\right)-\sum_{k=1}^{m} \frac{w_{k}}{W_{m}} f\left(\left|A_{k}-\sum_{j=1}^{m} \frac{w_{j}}{W_{m}} A_{j}\right|\right),
$$

which proves the desired result in (3.1). The particular case follows by setting $w_{k}=1$ for all $k=1, \cdots, n$ so that $W_{n}=n$.

Remark 2. The result in Lemma 7 was proved by Mond \& Pečarić in [28] for all operator convex functions and all bounded selfdjoint operators whose spectra contained in I. Therefore, in case $f$ is positive the inequality (3.1) might be considered as a respective extension and new refinement of that result proved in [28].

Theorem 3. Let $f: I \rightarrow \mathbb{R}$ be a real-valued continuous function. Let $\left(A_{1}, \cdots, A_{n}\right)$ be an n-tuple of positive of a Hilbert space $\mathscr{H}$ with spectra contained in $I$. Then the following conditions are equivalent:

(1) $f$ is operator superquadratic function.

(2) The inequality

$$
f\left(\sum_{k=1}^{n} C_{k}^{*} A_{k} C_{k}\right) \leq \sum_{k=1}^{n} C_{k}^{*} f\left(A_{k}\right) C_{k}-\sum_{k=1}^{n} C_{k}^{*} f\left(\left|A_{k}-\sum_{j=1}^{n} C_{j}^{*} A_{j} C_{j}\right|\right) C_{k}
$$

holds for every $n$-tuple $\left(C_{1}, \cdots, C_{n}\right)$ of operators on $\quad \mathscr{H}$ that satisfy the condition $\sum_{k=1}^{n} C_{k}^{*} C_{k}=1$.

(3) The inequality

$$
f\left(\sum_{k=1}^{n} P_{k} A_{k} P_{k}\right) \leq \sum_{k=1}^{n} P_{k} f\left(A_{k}\right) P_{k}-\sum_{k=1}^{n} P_{k} f\left(\left|A_{k}-\sum_{j=1}^{n} P_{j} A_{j} P_{j}\right|\right) P_{k}
$$

holds for every $n$-tuple $\left(P_{1}, \cdots, P_{n}\right)$ of projections on $\mathscr{H}$ with $\sum_{k=1}^{n} P_{k}=1$. 
Proof. (1) $\Rightarrow(2)$. We say that $C=\left(C_{1}, \cdots, C_{n}\right)$ is a unitary column if there is a unitary $n \times n$ operator matrix $U=\left(u_{i j}\right)$, one of whose columns is $\left(C_{1}, \cdots, C_{n}\right)$. Thus, $u_{i j}=C_{i}$ for some $j$ and all $i$. Assume that we are given a unitary $n$-column $\left(C_{1}, \cdots, C_{n}\right)$, and choose a unitary $U_{n}=\left(u_{i j}\right)$ in $\mathscr{B}\left(\mathscr{H}^{n}\right)$ such that $u_{k n}=C_{k}$. Let $E=\operatorname{diag}\left(\xi, \xi^{2}, \cdots, \xi^{n-1}, 1\right)$ as in Lemma 4 and put $X=\operatorname{diag}\left(A_{1}, \cdots, A_{n}\right)$, both regarded as elements in $\mathscr{B}\left(\mathscr{H}^{n}\right)$. Thus, using the spectral decomposition theorem, we have

$$
f\left(\sum_{k=1}^{n} C_{k}^{*} A_{k} C_{k}\right)=f\left(\left(U_{n}^{*} X U_{n}\right)_{n n}\right)=f\left(\left(\frac{1}{n} \sum_{k=1}^{n} E^{-k} U_{k}^{*} X U_{k} E^{k}\right)_{n n}\right) .
$$

We note that since $f\left(\operatorname{diag}\left(y_{1}, \cdots, y_{n}\right)\right)=\operatorname{diag}\left(f\left(y_{1}\right), \cdots, f\left(y_{n}\right)\right)$, then

$$
f\left(y_{n}\right)=f\left(\operatorname{diag}\left(y_{1}, \cdots, y_{n}\right)\right)_{n n}
$$

Using the above facts taking into account Lemmas $4-7$ together with the inequality (3.2), thus the operator superquadraticity of $f$, implies that

$$
\begin{aligned}
f\left(\sum_{k=1}^{n} C_{k}^{*} A_{k} C_{k}\right)= & f\left(\left(\frac{1}{n} \sum_{k=1}^{n} E^{-k} U_{k}^{*} X U_{k} E^{k}\right)_{n n}\right) \\
= & \left(f\left(\frac{1}{n} \sum_{k=1}^{n} E^{-k} U_{k}^{*} X U_{k} E^{k}\right)\right)_{n n} \\
\leq & \left(\frac{1}{n} \sum_{k=1}^{n} f\left(E^{-k} U_{k}^{*} X U_{k} E^{k}\right)\right)_{n n} \\
& -\left(\frac{1}{n} \sum_{k=1}^{n} f\left(\left|E^{-k} U_{k}^{*} X U_{k} E^{k}-\frac{1}{n} \sum_{j=1}^{n} E^{-j} U_{j}^{*} X U_{j} E^{j}\right|\right)\right)_{n n} \\
= & \left(\frac{1}{n} \sum_{k=1}^{n} E^{-k} U_{k}^{*} f(X) U_{k} E^{k}\right)_{n n} \\
& -\left(\frac{1}{n} \sum_{k=1}^{n} f\left(E^{-k} U_{k}^{*}\left|X-\frac{1}{n} \sum_{j=1}^{n} E^{-j} U_{j}^{*} X U_{j} E^{j}\right| U_{k} E^{k}\right)\right)_{n n} \\
= & \left(\frac{1}{n} \sum_{k=1}^{n} E^{-k} U_{k}^{*} f(X) U_{k} E^{k}\right)_{n n} \\
& -\left(\frac{1}{n} \sum_{k=1}^{n} E^{-k} U_{k}^{*} f\left(\left|X-\frac{1}{n} \sum_{j=1}^{n} E^{-j} U_{j}^{*} X U_{j} E^{j}\right|\right) U_{k} E^{k}\right)_{n n} \\
= & \left(U_{n}^{*} f(X) U_{n}\right)_{n n}-\left(U_{n}^{*} f\left(\left|X-U_{n}^{*} X U_{n}\right|\right) U_{n}\right)_{n n} \\
= & \sum_{k=1}^{n} C_{k}^{*} f\left(A_{k}\right) C_{k}-\sum_{k=1}^{n} C_{k}^{*} f\left(\left|A_{k}-\sum_{j=1}^{n} C_{j}^{*} A_{j} C_{j}\right| C_{k} .\right.
\end{aligned}
$$

It remains to mention that, when the column is just unital, we extend it to the unitary $(n+1)$-column $\left(C_{1}, \cdots, C_{n}, 0\right)$ and choose $A_{n+1}$ arbitrarily, but with spectrum in $I$, (see [8]). By the first part of the proof 
we therefore have

$$
\begin{aligned}
f\left(\sum_{k=1}^{n} C_{k}^{*} A_{k} C_{k}\right) & =f\left(\sum_{k=1}^{n+1} C_{k}^{*} A_{k} C_{k}\right) \\
& \leq \sum_{k=1}^{n+1} C_{k}^{*} f\left(A_{k}\right) C_{k}-\sum_{k=1}^{n+1} C_{k}^{*} f\left(\left|A_{k}-\sum_{j=1}^{n+1} C_{j}^{*} A_{j} C_{j}\right|\right) C_{k} \\
& =\sum_{k=1}^{n} C_{k}^{*} f\left(A_{k}\right) C_{k}-\sum_{k=1}^{n} C_{k}^{*} f\left(\left|A_{k}-\sum_{j=1}^{n} C_{j}^{*} A_{j} C_{j}\right|\right) C_{k}
\end{aligned}
$$

and thus the proof of the statement (2) is completely established.

$(2) \Rightarrow(3)$. Hold.

$(3) \Rightarrow(1)$. Let $A$ and $B$ be positive and bounded linear operators with spectra in $I$ and $0 \leq \lambda \leq 1$.

Consider

$$
\begin{gathered}
X=\left(\begin{array}{cc}
A & 0 \\
0 & B
\end{array}\right), P=\left(\begin{array}{cc}
1_{H} & 0 \\
0 & 0
\end{array}\right), Q=1_{H \otimes H}-P, \\
C=\left(\begin{array}{cc}
\sqrt{\lambda} & -\sqrt{1-\lambda} \\
\sqrt{1-\lambda} & \sqrt{\lambda}
\end{array}\right), \quad \text { and } \quad D=\left(\begin{array}{cc}
\sqrt{1-\lambda} & -\sqrt{\lambda} \\
\sqrt{\lambda} & \sqrt{1-\lambda}
\end{array}\right) .
\end{gathered}
$$

Then $C$ and $D$ are unitary operators on $H \oplus H$. We have

$$
\begin{aligned}
& C^{*} X C=\left(\begin{array}{cc}
\lambda A+(1-\lambda) B & 0 \\
0 & (1-\lambda) A+\lambda B
\end{array}\right) \\
& D^{*} X D=\left(\begin{array}{cc}
(1-\lambda) A+\lambda B & 0 \\
0 & \lambda A+(1-\lambda) B
\end{array}\right) \\
& P C^{*} X C P=\left(\begin{array}{cc}
\lambda A+(1-\lambda) B & 0 \\
0 & 0
\end{array}\right) \quad \text { and } \quad Q D^{*} X D Q=\left(\begin{array}{cc}
0 & 0 \\
0 & (1-\lambda) A+\lambda B
\end{array}\right) .
\end{aligned}
$$

Thus, we have

$$
\begin{aligned}
& f\left(\begin{array}{cc}
\lambda A+(1-\lambda) B & 0 \\
0 & (1-\lambda) A+\lambda B
\end{array}\right) \\
& =\left(\begin{array}{cc}
f(\lambda A+(1-\lambda) B) & 0 \\
0 & f((1-\lambda) A+\lambda B)
\end{array}\right) \\
& =f\left(P C^{*} X C P+Q D^{*} X D Q\right) \\
& \leq P f\left(C^{*} X C\right) P-P f\left(\left|C^{*} X C-P C^{*} X C P-Q D^{*} X D Q\right|\right) P \\
& +Q f\left(D^{*} X D\right) Q-Q f\left(\left|D^{*} X D-P C^{*} X C P-Q D^{*} X D Q\right|\right) Q \\
& =P C^{*} f(X) C P-P f\left(\left|C^{*} X C-P C^{*} X C P-Q D^{*} X D Q\right|\right) P \\
& +Q D^{*} f(X) D Q-Q f\left(\left|D^{*} X D-P C^{*} X C P-Q D^{*} X D Q\right|\right) Q \\
& =\left(\begin{array}{cc}
\lambda f(A)+(1-\lambda) f(B) & 0 \\
0 & (1-\lambda) f(A)+\lambda f(B)
\end{array}\right) \\
& -\left(\begin{array}{cc}
\lambda f((1-\lambda)|A-B|)+(1-\lambda) f(\lambda|A-B|) & 0 \\
0 & (1-\lambda) f(\lambda|A-B|)+\lambda f((1-\lambda)|A-B|)
\end{array}\right) .
\end{aligned}
$$

Hence, $f$ is operator superquadratic on $I$ by seeing the $(1,1)$-components.

Remark 3. An operator convex version of Theorem 3 were proved by Hansen $\mathscr{G}$ Pedersen in [18]. Therefore, in case $f$ is positive the inequality (3.3) could be considered as a new refinement of that result proved in [18].

A refinement of the classical Jensen's inequality (1.9) could be elaborated as follows: 
Corollary 2. Let $f: I \rightarrow \mathbb{R}$ be a real-valued continuous function. Let $A$ be a positive operator of a Hilbert space $\mathscr{H}$ with spectra contained in $I$. If $f$ is an operator superquadratic function, then the inequality

$$
f\left(C^{*} A C\right) \leq C^{*} f(A) C-C^{*} f\left(\left|A-C^{*} A C\right|\right) C
$$

holds for every operator $C$ on $\mathscr{H}$ that satisfy the condition $C^{*} C=1$.

Proof. Follows from Theorem 3 by setting $n=1$.

Remark 4. Let $f: I \rightarrow \mathbb{R}$ be a real-valued continuous function. Let $A$ be a positive operator of a Hilbert space $\mathscr{H}$ with spectra contained in $I$. If $f$ is an operator subquadratic function, then the inequality

$$
f\left(C^{*} A C\right) \geq C^{*} f(A) C-C^{*} f\left(\left|A-C^{*} A C\right|\right) C
$$

holds for every operator $C$ on $\mathscr{H}$ that satisfy the condition $C^{*} C=1$. Furthermore, by applying the subquadratic function $f(t)=t^{r}, t>0(r \in[0,1])$, then we have

$$
\left(C^{*} A C\right)^{r} \geq C^{*} A^{r} C-C^{*}\left|A-C^{*} A C\right|^{r} C
$$

for all $r \in[0,1]$.

A generalization of (3.5) (also, (1.7) and (1.8)) for any positive unital linear map between two Hilbert spaces having the same dimension is embodied in the following result.

Theorem 4. Let $\mathscr{H}, \mathscr{K}$ be two Hilbert spaces such that $\operatorname{dim} \mathscr{H}=\operatorname{dim} \mathscr{K} . \quad$ Let $f: J \rightarrow \mathbb{R}$ be a real-valued continuous function. Let $A$ be a positive of a Hilbert space $\mathscr{H}$ with spectra contained in $J$, and consider $\Phi: \mathscr{B}(\mathscr{H}) \rightarrow \mathscr{B}(\mathscr{K})$ be a positive unital linear map. If $f$ is operator superquadratic function, then the inequality

$$
f(\Phi(A)) \leq \Phi(f(A))-\Phi(f(|A-\Phi(A)|))
$$

holds. If $f$ is operator subquadratic, then the inequality (3.6) is reversed. Thus, the following refinement of $(1.7)$

is valid.

$$
\Phi^{2}(A) \leq \Phi\left(A^{2}\right)-\Phi\left(|A-\Phi(A)|^{2}\right)
$$

Proof. Let $A \in \mathscr{B}(\mathscr{H})$ be positive. Assume that $\mathcal{A}$ is the $C^{*}$-subalgebra of $\mathscr{B}(\mathscr{H})$ generated by $A$ and $1 \mathscr{H}$. Without loss of generality, we may assume that $\Phi$ is defined on $\mathcal{A}$. Since every unital positive linear map on a commutative $C^{*}$-algebra is completely positive. It follows that $\Phi$ is completely positive. So there exists (by Stinespring's theorem [29]), some isometry $V: \mathscr{H} \rightarrow \mathscr{K}$; and a unital $*$-homomorphism $\rho$ from $\mathcal{A}$ into the $C^{*}$-algebra $\mathscr{B}(\mathscr{H})$ such that $\Phi(A)=V^{*} \rho(A) V$. Clearly, $f(\rho(A))=\rho(f(A))$, for all continuous function $f$. Thus,

$$
\begin{aligned}
f(\Phi(A)) & =f(P \rho(A) P) \\
& \leq P f(\rho(A)) P-P f(|\rho(A-P \rho(A) P)|) P \quad(\text { by }(3.4) \text { with } n=1) \\
& =P \rho(f(A)) P-P \rho(f(|A-\Phi(A)|)) P \\
& =\Phi(f(A))-\Phi(f(|A-\Phi(A)|)) .
\end{aligned}
$$

which proves the required inequality. The last inequality holds by applying (3.6) to the superquaratic function $f(t)=t^{2}, \forall t>0$.

The inequality (3.6) can be embodied in multiple versions as stated in the following result.

Corollary 3. Let $\mathscr{H}, \mathscr{K} \quad$ be two Hilbert spaces such that $\operatorname{dim} \mathscr{H}=\operatorname{dim} \mathscr{K} . \quad$ Let $f:[0, \infty) \rightarrow \mathbb{R}$ be a real-valued continuous function. and consider $\Phi_{k}: \mathscr{B}(\mathscr{H}) \rightarrow \mathscr{B}(\mathscr{K})(k=1, \cdots, n)$ be a positive linear mappings with $\sum_{k=1}^{n} \Phi_{k}\left(1_{\mathscr{H}}\right)=1_{\mathscr{K}}$. Then, $f$ is operator superquadratic function if and only if

$$
f\left(\sum_{k=1}^{n} \Phi_{k}\left(A_{k}\right)\right) \leq \sum_{k=1}^{n} \Phi_{k}\left(f\left(A_{k}\right)\right)-\sum_{k=1}^{n} \Phi_{k}\left(f\left(\left|A_{k}-\sum_{j=1}^{n} \Phi_{j}\left(A_{j}\right)\right|\right)\right)
$$

for all positive operators $A_{1} \cdots, A_{n}$ in $\mathscr{B}(\mathscr{H})$. 
Proof. The proof is obvious.

\section{REFERENCES}

[1] S. Abramovich, G. Jamesion and G. Sinnamon, Inequalities for averages of convex and superquadratic functions, J. Inequ. Pure Appl. Math. , 5 (4) (2004), Article 91.

[2] S. Abramovich, G. Jameson and G. Sinnamon, Refining Jensen's inequality, Bull. Math. Soc. Sci. Math. Roumanie, 47 (2004), 3-14.

[3] S. Abramovich, J. Barić and J. Pečarič, A new proof of an inequality of Bohr for Hilbert space operators, Linear Algebra Appl., 430 (2009), 1432-1435.

[4] S. Abramovich, On superquadraticity, J. Math. Inequal. , 3 (3) (2009), 329-339.

[5] S. Abramovich, S. Ivelić and J. Pečarić, Improvement of Jensen-Steffensen's inequality for superquadratic functions, Banach J. Math. Anal., 4 (1) (2010), 146-158.

[6] R.P. Agarwal and S.S. Dragomir, A survey of Jensen type inequalities for functions of selfadjoint operators in Hilbert spaces, Comput. Math. Appl., 59 (2010), 3785-3812.

[7] M.W. Alomari, Operator Popviciu's inequality for superquadratic and convex functions of selfadjoint operators in Hilbert spaces, Advan. Pure Appl. Math., accepted

[8] H. Araki and F. Hansen, Jensen's operator inequality for functions of several variables, Proc. Amer. Math. Soc., 128 (2000), 2075-2084.

[9] R. Bhatia and R. Sharma, Some inequalities for positive linear maps, Linear Algebra Appl., 436 (2012), $1562-1571$.

[10] R. Bhatia, Matrix Analysis, Springer, 1996.

[11] J. Barić, A. Matković and J. Pečarić, A variant of the Jensen-Mercer operator inequality for superquadratic functions, Math. Comput. Modelling, 51 (2010) 1230-1239.

[12] S. Banić, J. Pečarić and S. Varošanec, Superquadratic functions and refinements of some classical inequalities, J. Korean Math. Soc., 45, (2) (2008), 513-525.

[13] J. Bendat and S. Sherman, Monotone and convex operator functions, Trans. Amer. Math. Soc., 79 (1955), 58-71.

[14] P. Chansangiam, A Survey on Operator Monotonicity, Operator Convexity, and Operator Means, Internat. J. Anal., Volume 2015, Article ID 649839, 8 pages

[15] M.D. Choi, A Schwarz inequality for positive linear maps on $C^{*}$-algebras, Illinois J. Math., 18 (1974), 565-574.

[16] J.C. Davis, A Schwarz inequality for convex operator functions, Proc. Amer. Math. Soc., 8 (1957) 42-44.

[17] T. Furuta, J. Mićić, J. Pečarić and Y. Seo, Mond-Pečarić method in operator inequalities. Inequalities for bounded selfadjoint operators on a Hilbert space, Element, Zagreb, 2005.

[18] F. Hansen G.K. Pedersen, Jenssen's operator inequality, Bull. London Math. Soc., 35 (2003) 553-564.

[19] F. Hansen and G.K. Pedersen, Jensen's inequality for operators and Löwner's theorem', Math. Ann., 258 (1982), $229-241$.

[20] J. Jensen, Sur les fonctions convexes et les inégalités entre les valeurs moyennes, Acta Math., 30 (1906), $175-193$.

[21] R.V. Kadison, A generalized Schwarz inequality and algebraic invariants for operator algebras, Ann. Math., 56 (1952), 494-503.

[22] M. Krnić, N. Lovrinčević, J. Pečarić and J. Perić, Superadditivity and monotonicity of the Jensen-type functionals: New Methods for improving the Jensen-type Inequalities in Real and in Operator Cases, Element, Zagreb, 2016.

[23] F. Kraus, Über konvexe Matrixfunktionen, Mathematische Zeitschrift, 41 (1) (1936), 18-42.

[24] M. Kian, Operator Jensen inequality for superquadratic functions, Linear Algebra and its Applications, 456, (2014), 82-87.

[25] M. Kian and S.S. Dragomir, Inequalities involving superquadratic functions and operators, Mediterr. J. Math., 11 (4)(2014), $1205-1214$.

[26] C.P. Niculescu, L.E. Persson, Convex Functions and Their Applications. A Contemporary Approach, CMS Books Math., vol. 23, Springer-Verlag, New York, 2006.

[27] D.S. Mitrinović, J. Pečarić and A.M. Fink, Classical and New Inequalities in Analysis, Kluwer Academic, Dordrecht, 1993.

[28] B. Mond and Pečarić, Convex inequalities in Hilbert space, Houston J. Math., 19 (1993), 405-420.

[29] W. F. Stinespring, Positive functions on $C^{*}$-algebras, Proc. Amer. Math. Soc., 6 (1955), 211-216.

Department of Mathematics, Faculty of Science and Information Technology, Jadara University, P.O. Box 733, Irbid, P.C. 21110, Jordan.

Email address: mwomath@gmail.com 